\title{
Layer resolved magnetization dynamics in coupled magnetic films using time-resolved $x$-ray magnetic circular dichroism with continuous wave excitation
}

\author{
T. Martin, ${ }^{1, a)}$ G. Woltersdorf, ${ }^{1}$ C. Stamm, ${ }^{2}$ H. A. Dürr, ${ }^{2}$ R. Mattheis, ${ }^{3}$ C. H. Back, ${ }^{1}$ and \\ G. Bayreuther ${ }^{1}$ \\ ${ }^{1}$ Institut für Experimentelle und Angewandte Physik, Universität Regensburg, Universitätsstraße 31, \\ 93040 Regensburg, Germany \\ ${ }^{2}$ Berliner Elektronenspeicherring-Gesellschaft für Synchrotronstrahlung mbH, Albert-Einstein-Straße 15, \\ 12489 Berlin, Germany \\ ${ }^{3}$ Institut für Photonische Technologien e.V., Albert-Einstein-Straße 9, 07745 Jena, Germany
}

(Presented 13 November 2008; received 15 September 2008; accepted 12 November 2008; published online 5 March 2009)

Time-resolved x-ray magnetic circular dichroism was used to investigate ferromagnetically coupled $\mathrm{CoFe} / \mathrm{Ru} / \mathrm{NiFe}$ bilayers. The magnetization dynamics was driven by a continuous wave excitation. The precessional motion of the individual layers was detected separately by tuning the x-ray photon energy to the $L_{3}$ absorption edge of either $\mathrm{Ni}$ or Co. Using two different waveguide stack geometries in-phase and antiphase excitation could be selected showing its effect in the measured precessional signal of the individual layers. In exchange-coupled bilayer two precessional modes were observed for each layer. The relative phase angles of the magnetic response between the two layers were found to be $8^{\circ}$ and $133^{\circ}$, for the two modes revealed their quasiacoustic and quasioptic character.

(C) 2009 American Institute of Physics. [DOI: 10.1063/1.3068650]

Interlayer exchange-coupled magnetic layers are important building blocks of magnetoelectronic devices. They are used in magnetic random access memories as well as in sensors based on the giant magnetoresistance effect. The ever increasing transfer speeds in such devices focus the interest on the dynamic behavior of such coupled bilayers. The element specificity of $\mathrm{x}$-ray magnetic circular dichroism (XMCD) gives the unique opportunity to observe the magnetization dynamics in such coupled bilayers in a layer resolved way giving information on the individual phase and amplitude of the precessional motion.

Previously time-resolved XMCD (TR-XMCD) experiments with pulsed excitation on coupled films have been reported. ${ }^{1}$ Here we discuss TR-XMCD experiments with continuous microwave excitation on the same samples. The presented measurement method typically allows for roughly 50 times shorter measuring time compared to pulsed excitation due to the better utilization of the available x-ray intensity. A similar measurement method was first reported by Arena et $a l . l^{2,3}$ Time-averaged FMR XMCD measurement methods are discussed in Refs. 4-6.

The investigated $\mathrm{Ta}(5 \mathrm{~nm}) / \mathrm{Co}_{90} \mathrm{Fe}_{10}(20 \mathrm{~nm}) / \mathrm{Ru}(t) /$ $\mathrm{Ni}_{81} \mathrm{Fe}_{19}(20 \mathrm{~nm}) / \operatorname{metal}(d)$ film systems were prepared by sputter deposition. By variation of the Ru thickness $t$ different coupling constants were achieved. Here we investigate three samples with different coupling strengths: sample 1 uncoupled $(t=10 \mathrm{~nm})$, sample 2 weak ferromagnetic coupling $(t=2.8 \mathrm{~nm})$, and sample 3 ferromagnetic coupling $(t$ $=1.4 \mathrm{~nm}$ ). The film system was grown on a $100 \mathrm{~nm}$ thick $\mathrm{Si}_{3} \mathrm{~N}_{4}$-membrane supported by a silicon frame in order to

${ }^{a)}$ Electronic mail: tobias.martin@physik.uni-regensburg.de. allow transmission of $x$ rays through the film system. The film system itself was patterned as a coplanar waveguide by photolithography and liftoff technique. By variation of the thickness $d$ and the material of the capping metal layer, the cross-sectional current distribution through the central conductor of the coplanar waveguide was changed. With a 100 $\mathrm{nm}$ thick $\mathrm{Cu}$ capping layer conducting more than $90 \%$ of the current, a parallel rf-magnetic field is expected in both magnetic layers. On the other hand, by replacing the thick $\mathrm{Cu}$ capping layer by a $5 \mathrm{~nm}$ thin $\mathrm{Ta}$ or $\mathrm{Al}$ capping layer, the cross-sectional current flow through the film system is approximately symmetric, thus leading to antiparallel magnetic excitation fields in the individual magnetic layers. A detailed discussion of the sample preparation is given in Ref. 1.

The samples were mounted in an ultrahigh vacuum chamber equipped with broadband rf connectors. The magnetic excitation field created by the coplanar waveguide is oriented along the $y$-direction [see Fig. 1(a)]. An electromagnet (not shown) is installed on the sample holder producing a dc magnetic bias-field $H_{b}$ along the $x$-direction. Continuous microwave excitation leads to a forced precession of the magnetization around $H_{b}$. This precession is elliptic due to the demagnetizing field of the magnetic film. The $\mathrm{x}$-ray beam is oriented at an angle of $35^{\circ}$ with respect to the film normal ( $z$-direction). Therefore a small deviation of the magnetization direction from the equilibrium $x$-axis changes the absorption of circularly polarized $\mathrm{x}$ rays due to XMCD. This effect is proportional to the sine of the angle of the magnetization with respect to the $x$-axis, which can be calculated if an appropriate reference measurement is performed. ${ }^{1}$ The transmitted x-ray intensity is detected with a photodiode.

At the electron synchrotron BESSY x-ray bunches with a full width at half maximum of approximately $70 \mathrm{ps}$ are de- 


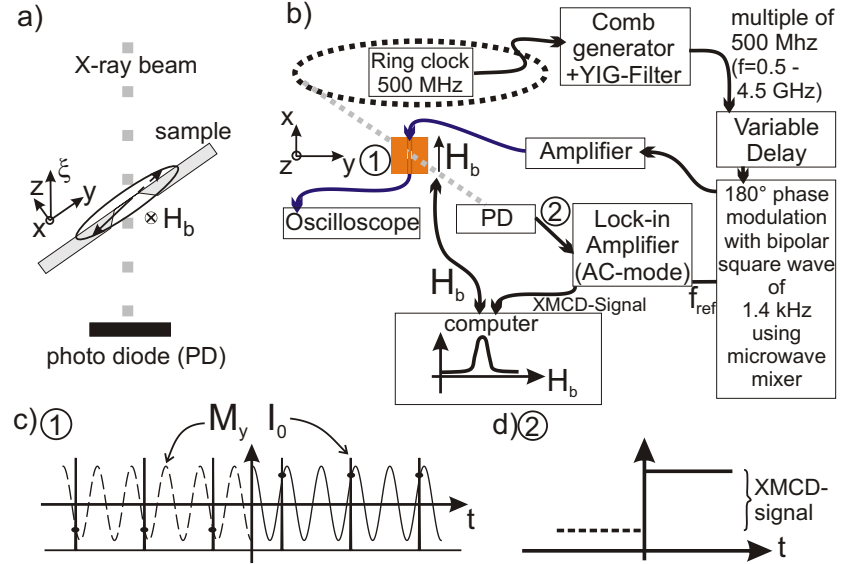

FIG. 1. (Color online) (a) Setup; (b) schematics of the measurement method. Signal at the points 1 (c) and 2 (d) in (b) (not to scale).

livered at a repetition rate of $500 \mathrm{MHz}$. The X-ray energy can be tuned to the desired value using a monochromator. In the described experiment a synchronization between the x-ray bunches and the excitation signal is accomplished as shown in Fig. 1(b). The ring clock frequency of $500 \mathrm{MHz}$, which is synchronous with the $\mathrm{x}$-ray bunches is used to generate a frequency comb. One of the harmonics is selected by a yttrium iron garnet filter. The phase of the microwave signal can be adjusted by an electronic delay generator in 5 ps steps. In addition the microwave phase is modulated by $180^{\circ}$ at a frequency of $f_{\text {ref }}=1.4 \mathrm{kHz}$ using a mixer. This phase modulation allows lock-in technique to be used for the signal detection. For the experiments shown here a microwave power of up to $26 \mathrm{dBm}$ was delivered to the sample. Due to the synchronization of the exciting microwave signal and the $\mathrm{x}$-ray bunches, the magnetization is sampled at a given constant phase $\varphi$ or $180^{\circ}+\varphi$ due to the $180^{\circ}$ phase modulation of the excitation [Fig. 1(c)]. This leads to a rectangular effective intensity signal with a frequency of $1.4 \mathrm{kHz}$ at the photodiode [Fig. 1(d)], which averages the X-ray pulses arriving at 2 ns time separation. The amplitude of the rectangular intensity signal is proportional to the XMCD signal and thus a measure for the actual magnetization component $m_{y z}$ projected onto the X-ray beam axis $m_{\xi}$.

This excitation and detection scheme allows for two different measurement techniques to be applied. (1) By scanning the delay of the excitation signal with respect to the $\mathrm{x}$-ray pulse train, the temporal evolution of the observed quantity, which is proportional to $m_{\xi}$, is measured at constant bias field. This yields a sinusoidal signal of frequency given by the exciting microwaves, from which the amplitude and the phase of the dynamic magnetization component can be determined. In a magnetic bilayer the phase difference of the precession of both magnetizations can be determined precisely (see Fig. 3 inset). (2) By sweeping the bias field through the resonance condition of both layers, resonance curves are obtained. By adjusting the phase of the exciting microwaves to $90^{\circ}$ or $0^{\circ}$ with respect to the $\mathrm{x}$-ray bunches, the measured quantity $\left(\propto m_{\xi}\right)$ represents either the imaginary part or the real part (not shown here) of the dynamic magnetic susceptibility, respectively. From the resonance curves the resonance field and the linewidth can be obtained.

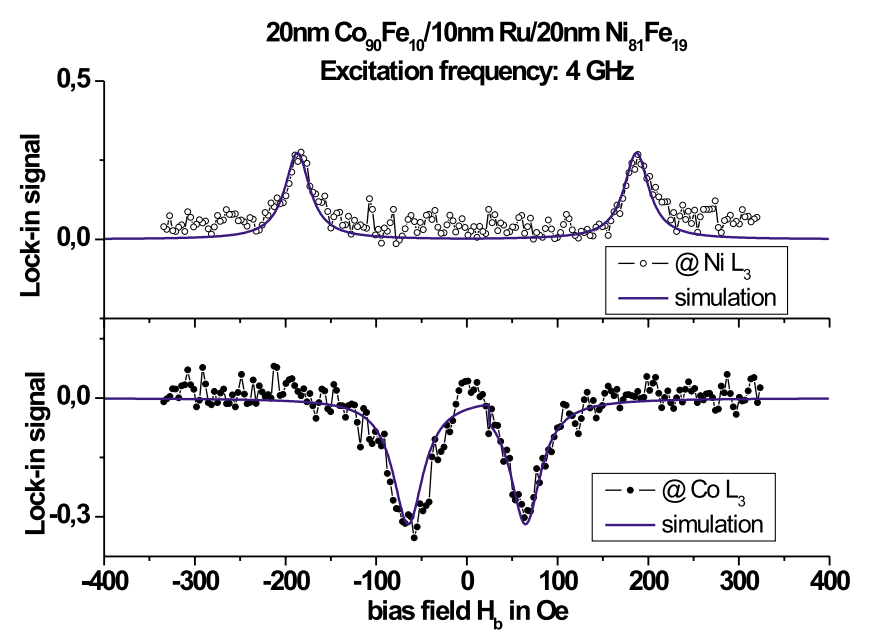

FIG. 2. (Color online) Bias-field dependence of the imaginary part of the dynamic magnetic susceptibility measured at the Ni $L_{3}$ and the $\mathrm{Co} L_{3}$ absorption edges of the $\mathrm{Co}_{90} \mathrm{Fe}_{10}(20 \mathrm{~nm}) / \mathrm{Ru}(10 \mathrm{~nm}) / \mathrm{Ni}_{81} \mathrm{Fe}_{19}(20 \mathrm{~nm}) /$ $\mathrm{Al}(5 \mathrm{~nm})$ film system, fitted with the simulation for a coupling strength of $0 \mu \mathrm{J} / \mathrm{m}^{2}$. Antiphase excitation; excitation frequency: $4 \mathrm{GHz}$.

The dynamic magnetic susceptibility, obtained in the experiments, can be calculated by solving a system of two coupled Landau-Lifshitz-Gilbert equations. It is important to include the damping term, in order to obtain realistic values for amplitude and phase. To do so the model used in Ref. 7 was extended accordingly. The results from bias-field dependent simulations were fitted to the experimental data. The different excitation geometries, e.g., in-phase and antiphase excitations, were also considered. The material specific variables used for the simulations were determined with conventional ferromagnetic resonance (FMR) measurements: saturation magnetization $M_{\mathrm{NiFe}}=(810 \pm 30) \times 10^{3} \mathrm{kA} / \mathrm{m}, M_{\mathrm{FeCo}}$ $=(1470 \pm 50) \times 10^{3} \mathrm{kA} / \mathrm{m}$, uniaxial anisotropy constants $K_{u, \mathrm{FeCo}}=2000 \pm 1000 \mathrm{~J} / \mathrm{m}^{3}$ and $K_{u, \mathrm{NiFe}}=600 \pm 400 \mathrm{~J} / \mathrm{m}^{3}$, damping parameters: $\quad \alpha_{\mathrm{NiFe}}=0.011 \pm 0.02, \quad \alpha_{\mathrm{CoFe}}$ $=0.013 \pm 0.03$.

Figure 2 shows the bias-field dependence of the measured XMCD signal, which is proportional to the imaginary part of the dynamic magnetic susceptibility for sample 1 . The resonance curves are obtained at the $\mathrm{Ni} L_{3}$ and $\mathrm{Co} L_{3}$ absorption edges of the sample $\mathrm{Co}_{90} \mathrm{Fe}_{10}(20 \mathrm{~nm}) / \mathrm{Ru}(10 \mathrm{~nm}) /$ $\mathrm{Ni}_{81} \mathrm{Fe}_{19}(20 \mathrm{~nm}) / \mathrm{Al}(5 \mathrm{~nm})$, thus corresponding to either magnetic layer. The sign of the measured signal is opposite for the individual layers due to the antiphase excitation. The excitation frequency is $4 \mathrm{GHz}$. Each magnetic layer has its individual resonance field. Within the given noise margin, no mutual influence of the layers is observed. The coupling strength $J$ was derived to be smaller than $20 \mu \mathrm{J} / \mathrm{m}^{2}$ by comparison with simulations while $J$ was varied. The fit shown in Fig. 2 corresponds to $J=0$. In this case the phase difference between the precession of both layers at the resonance of one layer is nearly $90^{\circ}$. The kink in the simulation data of the $\mathrm{Co}_{90} \mathrm{Fe}_{10}$-layer at approximately $25 \mathrm{Oe}$ is due to the magnetization reversal. Both, simulation and measurement of the imaginary part of the susceptibility as a function of field are shown for all three samples in Figs. 2-4.

Figure 3 shows the resonance curves for sample 2, $\mathrm{Co}_{90} \mathrm{Fe}_{10}(20 \mathrm{~nm}) / \mathrm{Ru}(2.8 \mathrm{~nm}) / \mathrm{Ni}_{81} \mathrm{Fe}_{19}(20 \mathrm{~nm}) / \mathrm{Ta}(5 \mathrm{~nm})$, 


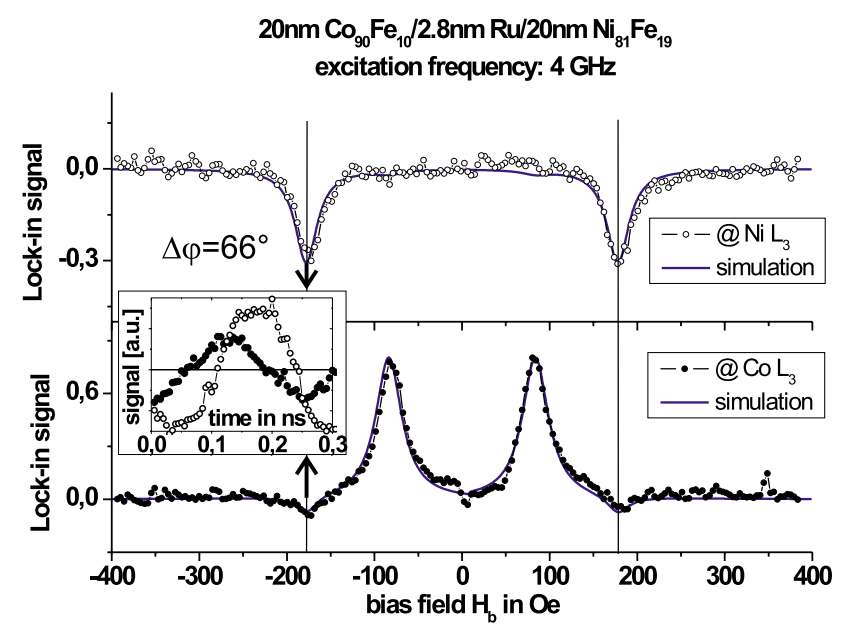

FIG. 3. (Color online) Bias-field dependence of the imaginary part of the dynamic magnetic susceptibility measured at the Ni $L_{3}$ and the Co $L_{3}$ absorption edges of the $\mathrm{Co}_{90} \mathrm{Fe}_{10}(20 \mathrm{~nm}) / \mathrm{Ru}(2.8 \mathrm{~nm}) / \mathrm{Ni}_{81} \mathrm{Fe}_{19}(20 \mathrm{~nm}) /$ $\mathrm{Ta}(5 \mathrm{~nm})$ film system at an excitation frequency of $4 \mathrm{GHz}$. The fit with the simulation gives a coupling strength of $15 \pm 3 \mu \mathrm{J} / \mathrm{m}^{2}$. The excitation happens antiphase. Inset: time-dependent measurement at the resonance field of the $\mathrm{Ni}_{81} \mathrm{Fe}_{19}$ layer, yielding a phase difference $\Delta \varphi$ of $66^{\circ} \pm 1^{\circ}$ between the precession signals of the $\mathrm{Ni}_{81} \mathrm{Fe}_{19}$ and $\mathrm{Co}_{90} \mathrm{Fe}_{10}$ layers.

a film system with weak ferromagnetic coupling, which is again excited antiphase at $4 \mathrm{GHz}$. Here a small influence of the $\mathrm{Ni}_{81} \mathrm{Fe}_{19}$ precession on the $\mathrm{Co}_{90} \mathrm{Fe}_{10}$ precession is visible as a "transferred" resonance peak at a field of 180 Oe. At the resonance field of the $\mathrm{Ni}_{81} \mathrm{Fe}_{19}$ layer a time-dependent measurement (see inset Fig. 3) results in a phase difference of $66^{\circ} \pm 1^{\circ}$. The influence of the $\mathrm{Co}_{90} \mathrm{Fe}_{10}$ on the $\mathrm{Ni}_{81} \mathrm{Fe}_{19}$ at 90 Oe is not visible in the experiment, but the simulation shows a tiny peak. The fit with the theoretical model is excellent and yields a coupling constant of $15 \pm 3 \mu \mathrm{J} / \mathrm{m}^{2}$. As the interlayer thickness is too large for an effective exchange coupling, the observed coupling is most probably of dipolar nature, although exchange coupling cannot be excluded. The determination of this small coupling constant was not possible with conventional methods such as standard FMR. This is an advantage of the presented method. ${ }^{2}$

Figure 4 shows the resonance curves of sample 3, a $\mathrm{Co}_{90} \mathrm{Fe}_{10}(20 \mathrm{~nm}) / \mathrm{Ru}(1.4 \mathrm{~nm}) / \mathrm{Ni}_{81} \mathrm{Fe}_{19}(20 \mathrm{~nm}) / \mathrm{Cu}(100 \mathrm{~nm})$ film system with ferromagnetic exchange coupling. Due to the thick overlayer both magnetic layers are excited in-phase $(f=4.5 \mathrm{GHz})$. From the fit to the resonance curve a coupling constant of $150 \pm 10 \mu \mathrm{J} / \mathrm{m}^{2}$ was deduced. Due to the stronger coupling compared to the sample 2 shown in Fig. 3, the mutual influence is more obvious. Both quasioptic and quasiacoustic modes are clearly observed with phase differences of $133^{\circ} \pm 1^{\circ}$ and $8^{\circ} \pm 1^{\circ}$, respectively.

In summary, layer resolved magnetization dynamics measurements of coupled magnetic bilayers were presented using TR-XMCD with continuous microwave excitation. Small coupling constants, not detectable with conventional measurement methods, were quantitatively determined by fitting the experimental data to the theoretical model. The unique layer resolved information on phase and amplitude of

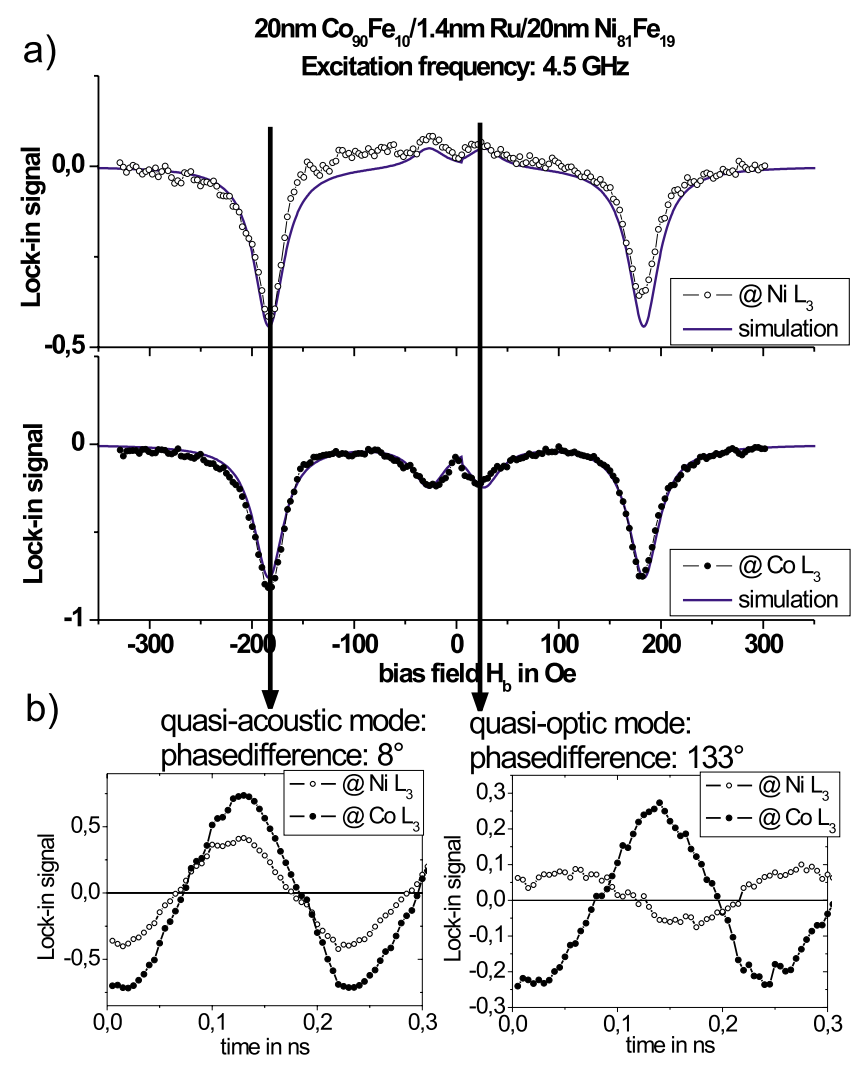

FIG. 4. (Color online) (a) Bias-field dependence of the imaginary part of the dynamic magnetic susceptibility measured at the Ni $L_{3}$ and the Co $L_{3}$ absorption edges of the $\mathrm{Co}_{90} \mathrm{Fe}_{10}(20 \mathrm{~nm}) / \mathrm{Ru}(1.4 \mathrm{~nm}) / \mathrm{Ni}_{81} \mathrm{Fe}_{19}(20 \mathrm{~nm}) /$ $\mathrm{Cu}(100 \mathrm{~nm})$ film system at an excitation frequency of $4.5 \mathrm{GHz}$. The fit with the simulation gives a coupling strength of $150 \pm 10 \mu \mathrm{J} / \mathrm{m}^{2}$. The excitation happens in phase. (b) Time-dependent measurement at the resonance field of quaisacoustic and quasioptic modes.

the dynamic magnetic susceptibility offered by the presented method will be of advantage for future investigations of other dynamic effects in magnetic bilayers, such as damping and the spin pumping effect. ${ }^{8}$

Support by the Deutsche Forschungsgemeinschaft DFG (Grant No. SPP1133) and BMBF (Grant No. 05 ES3XBA/5) is gratefully acknowledged. The authors thank T. Kachel for his support at the BESSY beamline and D. Weiss for the access to the cleanroom facilities.

${ }^{1}$ T. Martin, G. Woltersdorf, C. Stamm, H. A. Dürr, R. Mattheis, C. H. Back and G. Bayreuther, J. Appl. Phys. 103, 07B112 (2008).

${ }^{2}$ D. A. Arena, E. Vescovo, C.-C. Kao, Y. Guan, and W. E. Bailey, Phys. Rev. B 74, 064409 (2006).

${ }^{3}$ D. A. Arena, E. Vescovo, C.-C. Kao, Y. Guan, and W. E. Bailey, J. Appl. Phys. 101, 09C109 (2007).

${ }^{4}$ G. Boero, S. Rusponi, P. Bencok, R. S. Popovic, H. Brune, and P. Gambardella, Appl. Phys. Lett. 87, 152503 (2005).

${ }^{5}$ G. Boero, S. Mouaziz, S. Rusponi, P. Bencok, F. Nolting, S. Stepanow, and P. Gambardella, New J. Phys. 10, 013011 (2008).

${ }^{6}$ J. Goulon, A. Rogalev, F. Wilhelm, N. Jaouen, C. Goulon-Ginet, G. Goujon, J. Ben Youssef, and M. V. Indenbom, JETP Lett. 82, 696 (2005).

${ }^{7}$ M. Buchmeier, B. K. Kuanr, R. R. Gareev, D. E. Bürgler, and P. Grünberg, Phys. Rev. B 67, 184404 (2003).

${ }^{8}$ G. Woltersdorf, O. Mosendz, B. Heinrich, and C. H. Back, Phys. Rev. Lett. 99, 246603 (2007). 